CLNS-96/1409

hep-th/9605131

May 1996

\title{
The SO(32) Heterotic String on a K3 Surface
}

\author{
Paul S. Aspinwall \\ F.R. Newman Lab. of Nuclear Studies, \\ Cornell University, \\ Ithaca, NY 14853 \\ Mark Gross \\ Department of Mathematics, \\ Cornell University, \\ Ithaca, NY 14853
}

\begin{abstract}
The $\mathrm{SO}(32)$ heterotic string on a K3 surface is analyzed in terms of the dual theory of a type II string (or F-theory) on an elliptically fibred Calabi-Yau manifold. The results are in beautiful agreement with earlier work by Witten using very different methods. In particular, we find gauge groups of $\mathrm{SO}(32) \times \mathrm{Sp}(k)$ appearing at points in the moduli space identified with point-like instantons and see hypermultiplets in the $(\mathbf{3 2}, \mathbf{2 k})$ representation becoming massless at the same time. We also discuss some aspects of the $E_{8} \times E_{8}$ case.
\end{abstract}




\section{Introduction}

There are two heterotic superstring theories in ten dimensions which give rise to gauge groups $E_{8} \times E_{8}$ and $\mathrm{SO}(32)$ respectively. The simplest description of duality for these two theories looks quite different. The $E_{8} \times E_{8}$ string is believed to be dual to M-theory compactified on a finite line segment [1] whereas the $\mathrm{SO}(32)$ case is supposedly dual to a type I superstring [2, 3].

When the heterotic string is compactified on a circle (and therefore any manifold with a circle as a factor), the two theories become equivalent in the sense that the $E_{8} \times E_{8}$ string compactified on such a manifold is identical to the $\mathrm{SO}(32)$ case compactified on the same manifold with different moduli [4, 5]. It is interesting to ask whether this holds for other compactification spaces.

The case of the SO(32) string compactified on a K3 surface was studied in [6]. There it was shown that, although the unbroken gauge group was generically $\mathrm{SO}(8)$, by shrinking down some of the instantons one could achieve non-perturbative $\mathrm{Sp}(1)$ extra gauge group factors. ${ }^{1}$ If all 24 instantons are shrunk down then the gauge group is generically $\mathrm{SO}(32) \times \operatorname{Sp}(1)^{24}$. One may further enhance the gauge group by allowing these point-like instantons to coalesce. When $k$ instantons coalesce at a generic point in the K3 surface, the $\operatorname{Sp}(1)^{k}$ factor is expected to be replaced by $\mathrm{Sp}(k)$. Thus one may obtain $\mathrm{SO}(32) \times \mathrm{Sp}(24)$ as a gauge group in the case that all 24 instantons coalesce. The analysis of [6] and subsequent analysis (see, for example [7]) relied on this heterotic string's duality with the type I string, and $D$-brane technology.

The $E_{8} \times E_{8}$ string compactified on a K3 surface has recently been analyzed in [8, 9, 10]. In this case the analysis has been done in terms of M-theory or F-theory which, for purposes of this letter, should be thought of as limits of the type II superstring. In this case, when the instantons are shrunk down to zero size, no further gauge group appears but instead extra massless "tensor" multiplets appear which have no perturbative description in terms of the heterotic string.

It was conjectured in $[8]$ that the $\mathrm{SO}(32)$ heterotic string compactified on a $\mathrm{K} 3$ surface is the same as a particular case of the $E_{8} \times E_{8}$ heterotic string compactified on another K3 surface and so the situation for the two heterotic strings compactified on a K3 surface should be analogous to that of the circle above.

If this is the case we should be able to reproduce the analysis of the $\mathrm{SO}(32)$ string as in [6] in the same language as the $E_{8} \times E_{8}$ string was studied in [8, 9]. This is the goal of this letter. As well as providing yet another highly non-trivial test of string duality, we believe that the elliptic fibration language used to describe the heterotic string below will prove to be the more powerful (although this may be a question of taste).

While completing this work we became aware of [11 which has some overlap with the

\footnotetext{
${ }^{1}$ There is a closely-related ambiguity of a factor of 2 in counting supersymmetries in 5,6 or 7 dimensions and whether symplectic groups are denoted $\operatorname{Sp}(k)$ or $U S p(2 k)$. We will count quaternionically in both cases and so will be concerned with $N=1$ supersymmetry in 6 dimensions with gauge groups $\operatorname{containing} \operatorname{Sp}(k)$.
} 
results presented here.

\section{The Elliptic Fibration}

In this section we will review the analysis we require from [8, 9]. This was originally cast in the form of F-theory but here we choose to rephrase things to enable us to make some slightly stronger statements.

Begin with the widely-believed proposition that the type IIA string compactified on a K3 surface is equivalent to the $\left(E_{8} \times E_{8}\right.$ or $\left.\mathrm{SO}(32)\right)$ heterotic string compactified on a four-torus. Now use this duality fibre-wise as proposed in [12 to generate dual pairs in the form of a type IIA string compactified on a Calabi-Yau threefold and a heterotic string compactified on $\mathrm{K} 3 \times T^{2}$ of the type proposed in [13, 11]. The Calabi-Yau threefold is of the form of a K3fibration for such a scheme to work but this must be the case if the weakly-coupled heterotic string can be understood in terms of a perturbative sigma-model on the Calabi-Yau [15].

Since we need to handle various K3 surfaces and various fibrations, let us fix some notation. Let the type IIA string be compactified on the Calabi-Yau threefold $X$. We then have a fibration

$$
X \rightarrow \mathbb{P}^{1}(w),
$$

with generic K3 fibre, $S_{w}$, where $w$ is an affine coordinate on the base $\mathbb{P}^{1}$. String-string duality then replaces $S_{w}$ by $T_{w}^{4}$ for the heterotic string. Now let this $T_{w}^{4}$ factorize into $T_{0}^{2} \times T_{w}^{2}$, where $T_{0}^{2}$ is constant over $\mathbb{P}^{1}(w) . T_{w}^{2}$ is then the fibre for another fibration

$$
S_{H} \rightarrow \mathbb{P}^{1}(w)
$$

where $S_{H}$ is a K3 surface such that the heterotic string is compactified on $S_{H} \times T_{0}^{2}$

Now let the $T_{0}^{2}$ of the $S_{H} \times T_{0}^{2}$ acquire infinite area. This will decompactify the theory to the desired heterotic string compactified on the K3 surface, $S_{H}$. If we can understand in terms of the type II string what this large area limit of $T^{2}$ corresponds to, then we will have some type II picture of the heterotic string compactified on a K3 surface.

We thus regard the $\mathrm{N}=1$ theory in six dimensions of a heterotic string compactified on a K3 surface as dual to a special limit of a type IIA string theory compactified on some Calabi-Yau space, $X$. We need to isolate the $T_{0}^{2}$ degrees of freedom from the rest of the theory to allow it to go to infinite size. We thus demand that within $T_{w}^{4}$, we have a heterotic string compactified on $T_{0}^{2} \times T_{w}^{2}$, where the metric factorizes and no Wilson lines are wrapped around $T_{0}^{2}$. The Narain moduli space of a heterotic string on $T_{w}^{4}$ is thus restricted as

$$
\frac{O(4,20)}{O(4) \times O(20)} \supset \frac{O(2,18)}{O(2) \times O(18)} \times \frac{O(2,2)}{O(2) \times O(2)} .
$$

For the discrete identifications within this space we split the even self-dual lattice $\Gamma_{4,20}$ into two self-dual lattices $\Gamma_{2,18} \oplus \Gamma_{2,2}$. 
Such a factorization of the moduli space of heterotic strings on a torus is dual to a type IIA string compactified on a K3 surface which has a chosen algebraic type (see, for example, [16]). The factors of the moduli space then correspond to deformation of complex structure and the deformation of complexified Kähler form of the K3 surface. In the case at hand we want the K3 surface to have Picard lattice $\Gamma_{1,1}$. It follows that this K3 surface must be of the form of an elliptic fibration with a unique global section.

What we have shown therefore is that if we can decompactify the heterotic string to 6 dimensions as above, then $X$ must be a K3 fibration where the generic K3 fibre, $S_{w}$, is itself an elliptic fibration. We denote this fibration

$$
S_{w} \rightarrow \mathbb{P}_{w}^{1}(z),
$$

where the generic fibre is an elliptic curve $e(w, z)$. The notation $\mathbb{P}_{w}^{1}(z)$ means that the affine coordinate on this $\mathbb{P}^{1}$ is $z$, and there are a set of such $\mathbb{P}^{1}$ 's parametrized by $w$. The fact that this elliptic fibration has a unique section can then be used to rewrite $X$ itself as an elliptic fibration over some complex surface. This surface will be of the form of a fibration over $\mathbb{P}^{1}(w)$ with generic fibre $\mathbb{P}_{w}^{1}(z)$. That is, it is the Hirzebruch surface $\mathbf{F}_{n}$. It also follows that this elliptic fibration will have a section.

What exactly happens to $X$ as the $T^{2}$ in the heterotic string goes off to infinite size? Since the moduli space of the heterotic string on $T^{4}$ can be matched point-for-point with the moduli space of a type IIA string on a K3 surface [17], one can show that the effect on the K3 fibre within $X$ is as follows. The base $\mathbb{P}_{w}^{1}(z)$ blows up to infinite size but the area of the fibre $e(w, z)$ may be held constant. Also, if we want to make the dilaton of the six dimensional theory finite, the four-dimensional heterotic string must go to a weakly-coupled limit as $T_{0}^{2}$ gets larger. In terms of $X$, this means that the base $\mathbb{P}^{1}(w)$ of $X$ as a K3-fibration goes to infinite size 15.

At first sight it looks therefore as if $X$ grows in a way compatible with the elliptic fibration picture where the fibre's area remains constant but the base $\mathbf{F}_{n}$ goes to infinite volume. However, we need to make sure we are capturing the right degrees of freedom to describe six-dimensional physics. To do this, one may use M-theory type arguments along the lines of [2] or the F-theory arguments of [18] to see that what we should really do is to rescale the metric on $X$ so that it is the base $\mathbf{F}_{n}$ that remains finite sized but the elliptic fibre shrinks down to nothing.

The claim from [8] which we require that we have outlined above is thus the following. Any heterotic string compactified on a K3 surface is dual to a type IIA string theory compactified on a Calabi-Yau $X$, where $X$ is an elliptic fibration over $\mathbf{F}_{n}$ with a section. We also only consider degrees of freedom present when the fibre is shrunk down to zero size.

Hirzebruch surfaces $\mathbf{F}_{n}$ are classified by the number $n$. This is defined such that the self-intersection of the "exceptional" rational curve $C_{0} \subset \mathbf{F}_{n}$ is $-n$. We need to know how $n$ is determined by the heterotic string. This then determines what $X$ is. Recall that to obtain an anomaly-free theory, the heterotic string is compactified on a K3 surface along with a 
principal $G$-bundle, $E$, where $G$ is either $E_{8} \times E_{8}$ or $\mathrm{SO}(32)$ according to which heterotic string is used and $c_{2}(E)=24$. The homotopy class of a $G$-bundle over a K3 surface is defined by a map from $\pi_{3}(G)$ into $H^{4}(\mathrm{~K} 3)$ (see, for example, [19]). In the case of $G \cong \mathrm{SO}(32)$ we have that $\pi_{3} \cong \mathbb{Z}$ and this map is essentially fixed by the $c_{2}$ condition. In the case of $G \cong E_{8} \times E_{8}$ however, we have $\pi_{3} \cong \mathbb{Z} \oplus \mathbb{Z}$ and there remains an integer degree of freedom. The degree of freedom is the way that the 24 is divided between the two $E_{8}$ groups.

We thus expect that the $\mathrm{SO}(32)$ heterotic string determines a unique value for $n$ whereas the $E_{8} \times E_{8}$ string requires further specification of how the 24 is divided between the two factors. In [8, 9] it was argued that this split is $12-n$ and $12+n$ for the $E_{8} \times E_{8}$ string and it was conjectured that $n=4$ for the $\mathrm{SO}(32)$ string.

\section{Explicit Models}

$X$ is an elliptic fibration over $\mathbf{F}_{n}$ with $z$ the affine coordinate of the generic $\mathbb{P}^{1}$ fibre of $\mathbf{F}_{n}$ and $w$ the affine coordinate on the exceptional curve $C_{0}$ (i.e., the base). We may then write the elliptic fibration in Weierstrass form

$$
y^{2}=x^{3}+a(w, z) x+b(w, z) .
$$

The fact that $X$ is a Calabi-Yau space constrains $a$ and $b$ as follows. The canonical class of $\mathbf{F}_{n}$ is

$$
K_{\mathbf{F}_{n}}=-2\left[C_{0}\right]-(2+n)[f],
$$

where $\left[C_{0}\right]$ is the class of $C_{0}$ and $[f]$ is the class of the generic $\mathbb{P}^{1}$ fibre. Let $L$ be a line bundle such that $c_{1}(L)=-K_{\mathbf{F}_{n}}$. Then $a$ must be a section of $L^{\otimes 4}$ and $b$ must be a section of $L^{\otimes 6}$ [20].

To make direct contact with the heterotic string we look for ways in which enhanced gauge groups can appear. It is generally believed that this occurs in the type IIA string when ADE-like singularities are acquired along a curve in $X$ [21, 22, 16, 23]. In the first instance we will be interested in the case that these groups are understood perturbatively from the heterotic string. It was shown in [16] that this corresponds to the generic fibre of $X$ as a K3-fibration containing an ADE-like singularity.

The appearance of ADE-like singularities is well-understood in the case of elliptic surfaces and the possibilities are listed in [9]. The $E_{8} \times E_{8}$ case is relatively straight-forward as explained in [9] and we quickly repeat it here as a guide to the more subtle $\mathrm{SO}(32)$ case. Let us force $X$ to have an $E_{8} \times E_{8}$ singularity in every generic K3 fibre. This requires two II* fibres in Kodaira's notation. This may be done by specializing (5) to the form

$$
y^{2}=x^{3}+f(w) z^{4} x+g_{1}(w) z^{5}+g_{2}(w) z^{6}+g_{3}(w) z^{7} .
$$

It then follows immediately from the Calabi-Yau condition on $X$ above that $f(w)$ is of degree 8 in $w, g_{1}(w)$ is of degree $12-n, g_{2}(w)$ is of degree 12 and $g_{3}(w)$ is of degree $12+n$. 
At the $12-n$ zeros of $g_{1}(w)$ and the $12+n$ zeros of $g_{3}$ the singularities of the K3 fibre get worse. This is interpreted in the heterotic string language as follows [9]. To get the full $E_{8} \times E_{8}$ gauge group we go to an extreme case of the bundle $E$ which corresponds to 24 point-like instantons. Any smooth finite-sized instanton will break part of the gauge group. Thus, these worse singularities are simply the location of these 24 point-like instantons. The fact that $12-n$ points worsen one of the $\mathrm{II}^{*}$ fibres and $12+n$ worsen the other corresponds to the identification of $n$ as the way the 24 is split between the two $E_{8}$ factors as stated above.

It is perhaps worth noting that some duality magic is already at work here. The total instanton number of 24 was predicted by the Calabi-Yau condition on $X$. One may also show, using the methods of [9], that this condition also imposes the Calabi-Yau condition on the space the heterotic string lives on, namely that it must be K3.

In order that $X$ be a smooth manifold, the fibration (7) must be blown-up (in addition to the blow-ups of the II* fibres). In effect, the base $\mathbf{F}_{n}$ must be blown-up at 24 points. Given the results of the last section we may distinguish physically between blow-ups of the elliptic fibres and blow-ups of the base. In the four-dimensional picture of a type IIA string compactified on $X$, both blow-ups are deformations of the Kähler form on $X$ and are thus moduli living in four-dimensional vector multiplets. When we go to the six-dimensional picture, the fibre must be shrunk down to zero size. Thus the blow-ups of the fibre are not really moduli at all. The blow-ups of the base are still moduli however. Since a tensor multiplet in six dimensions contains a scalar and reduces to a vector multiplet in four dimensions; and a vector multiplet in six dimensions contains no scalars but also reduces to a vector multiplet in four dimensions the interpretation is clear when viewing $X$ as an elliptic fibration:

- Requiring a blow-up in the fibre indicates the appearance of a nonabelian gauge group.

- Requiring a blow-up of the base indicates a tensor multiplet.

Thus the compactification of the $E_{8} \times E_{8}$ string with zero sized instantons contains generically no further enhancement of the gauge group beyond what is know perturbatively, but will contain 24 massless scalars coming from tensor multiplets which have no perturbative interpretation for the heterotic string [10]. These extra moduli can be used to perform extremal transitions between heterotic strings of different values of $n$ [10, 8, 24].

Now we will try to do the same analysis for the $\mathrm{SO}(32)$ string. Firstly we ask that the theory (with the instantons shrunk down to points) has an unbroken perturbative gauge group of $\mathrm{SO}(32)$. This implies that the generic K3 fibre has an $\mathrm{I}_{12}^{*}$ fibre itself.

Let us assume that this happens when $z=0$. This means that (see, for example, [25])

$$
\begin{aligned}
a(w, z) & =z^{2} a_{0}(w, z), \\
b(w, z) & =z^{3} b_{0}(w, z), \\
\Delta=4 a(w, z)^{3}+27 b(w, z)^{2} & =z^{18} \Delta_{0},
\end{aligned}
$$


where $\Delta$ is the discriminant of the elliptic fibre and $a_{0}(w, z), b_{0}(w, z)$ and $\Delta_{0}$ are nonzero for $z=0$. We also subject $a(w, z)$ and $b(w, z)$ to the condition above that $X$ be a Calabi-Yau.

Fixing $w$, we expect to obtain a two parameter family of K3 surfaces. This is because our K3 surface has Picard number $20-2=18$. The problem is that, unlike the $E_{8} \times E_{8}$ case, the space of solutions is not connected. To fix which component of the moduli space is suitable for the $\mathrm{SO}(32)$ heterotic string, we require a further constraint.

The constraint we have not yet used is the fact that the $\mathrm{SO}(32)$ heterotic string is built using the even self-dual Barnes-Wall lattice $\Gamma_{16}$, as opposed to the root lattice of $\mathrm{SO}(32)$ which is not self-dual. Given the duality between the Picard lattice of the K3 surface and the set of winding/momenta modes of the heterotic string, this translates into the statement that the Picard lattice of the K3 surface, $S_{w}$, for a generic $w$, is self-dual.

The Picard lattice of a surface as an elliptic fibration has been analyzed in [26]. The result we require is as follows. Let the surface $S$ be an elliptic fibration and let $\Phi$ be the group of global sections, which we assume to be finite. Fix a section of $S$ and let $R$ be the sublattice of $\operatorname{Pic}(S)$ generated by components of fibres not meeting this section. We then have

$$
\operatorname{disc}(R)=|\Phi|^{2} \operatorname{disc}(\operatorname{Pic}(S))
$$

In the case of an $\mathrm{I}_{12}^{*}$ fibre we know that $R$ is the root lattice of $\mathrm{SO}(32)$ and thus has discriminant 4 . It follows that we require $|\Phi|=2$, i.e., $S_{w}$ must have two global sections as an elliptic fibration.

The Weierstrass form (5) has generically only one global section - at $x=y=\infty$. To force another global section we may write it in the more restricted form

$$
y^{2}=(x-\alpha(w, z))\left(x^{2}+\alpha(w, z) x+\beta(w, z)\right)
$$

which gives a section along $x=\alpha(w, z), y=0$. From (8) we see that $\alpha$ must have a zero of order 1 at $z=0$ and $\beta$ must vanish to order 2 . Now when we demand that $\Delta$ has a zero of order 18 we obtain a connected set of solutions as expected. The following represents a solution sufficiently generic for our purposes:

$$
\begin{aligned}
& \alpha(w, z)=B z^{4}+C z \\
& \beta(w, z)=A z^{8}-4 B C z^{5}-2 C^{2} z^{2} .
\end{aligned}
$$

The Calabi-Yau condition then imposes that $A$ is a polynomial of degree $8+4 n$ in $w, B$ is of degree $4+2 n$, and $C$ is of degree $4-n$. The discriminant on $\mathbf{F}_{n}$ takes the form

$$
\Delta=z^{18}\left(A+2 B^{2}\right)^{2}\left(\left(4 A-B^{2}\right) z^{6}-18 B C z^{3}-9 C^{2}\right) .
$$

Clearly from (12) we see the $\mathrm{I}_{12}^{*}$ fibres along $z=0$ generating the $\mathrm{SO}(32)$ gauge group. We also see that interesting things are going to happen at the $8+4 n$ zeros of $A+2 B^{2}$. 
Identifying these as the small instantons of the $\mathrm{SO}(32)$ heterotic string, we see independent evidence that we require $n=4$. In this case there will be 24 small instantons - precisely what is required for the $\mathrm{SO}(32)$ gauge symmetry to appear. From now on we will assume that $n=4$.

A remarkable agreement with the results of [6] occurs when we ask exactly what happens at the zeros of $A+2 B^{2}$. Consider a zero of $A+2 B^{2}$ of order $k$ in $w$. This will correspond to $k$ point-like instantons coalescing. The generic case will be $k=1$. The zero of the discriminant (12) will be of order $2 k$ in $w$ for any generic value of $z$. The terms $a(w, z)$ and $b(w, z)$ are generically nonzero. Thus according to [25], we have a curve of $\mathrm{I}_{2 k}$ fibres. As mentioned earlier, blow-ups in such a fibre correspond to extra gauge groups appearing. That is, each small instanton is generating a new nonperturbative contribution to the gauge group. At first sight, according to [9], the gauge group associated to $\mathrm{I}_{2 k}$ is $\mathrm{SU}(2 k)$. There is a subtlety which changes this however.

In the analysis of [16], where an enhanced gauge group appeared because of a degeneration of every generic K3 fibre, care was taken to restrict to the case where the Picard group of the K3 fibre was monodromy invariant as one moved around the base $\mathbb{P}^{1}$. In the case at hand, it turns out that the rational curves within the blow-up of the $\mathrm{I}_{2 k}$ fibre are not monodromy invariant as we move around the curve within $\mathbf{F}_{4}$ of fixed $w$. To obtain the enhanced gauge group that really appears, we require the monodromy invariant part of the apparent local gauge group over each point. The action of the monodromy on the blown-up fibre can be translated into an action on the Dynkin diagram of the simply-laced local gauge group. The required group is then the subgroup invariant under this outer automorphism. We can thus obtain non-simply-laced gauge groups in the type II setting. This is fairly analogous to the way such groups appeared in the heterotic string [27].

In the case at hand, there is a $\mathbb{Z}_{2}$ monodromy group which acts on the $\mathrm{I}_{2 k}$ fibre as shown in figure 1 (for the case $k=3$ ). This was demonstrated in [25]. Results for finding the invariant part of Lie algebras under outer automorphisms may be found in 28 for example. We see that the gauge group which appears when $k$ instantons coalesce is $\operatorname{Sp}(k)$ in complete agreement with [6].

\section{Hypermultiplets}

In [6] it was predicted that one should also obtain hypermultiplets in a $(\mathbf{3 2}, \mathbf{2} \boldsymbol{k})$ representation of $\mathrm{SO}(32) \times \mathrm{Sp}(k)$ when small instantons appear. It was suggested in 9] that the appearance of hypermultiplets was connected somehow to the collision of loci of bad elliptic fibres within the base $\mathbf{F}_{n}$. We will attempt to go some way to justifying these statements here.

First recall the "wrapping $p$-brane" of [29] and the way in which it applied to enhanced gauge symmetries of a type IIA string on a K3 surface. When rational curves in the K3 surface shrink down to zero size, extra massless particles appear whose charges fill out the 


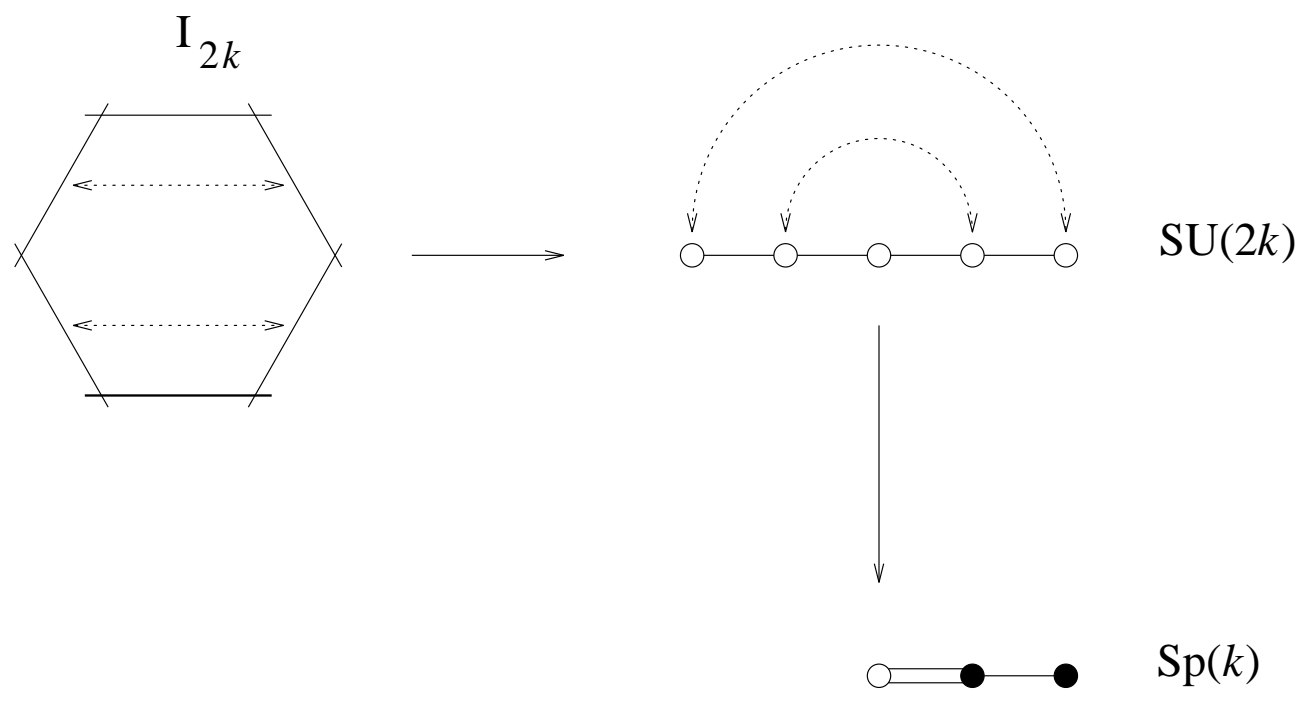

Figure 1: The generation of the gauge group $\operatorname{Sp}(k)$.

root lattice of the resulting nonabelian group. The charges are dictated by their interaction with the $U(1)$ vector fields which in turn can be measured by how their mass increases as moduli within these vector supermultiplets are switched on to Higgs away the enhanced gauge symmetry. The mass is given by the area which in turn is given by the Kähler form's value on the homology class of the rational curve. That is, the set of elements in $H_{2}$ of the rational curve classes around which the 2-branes wrap form the root lattice of the enhanced gauge group.

For the type IIA string compactified on $X$, the above picture happens along a whole curve of ADE-like singularities to produce an enhanced gauge group. At points along this curve, the singularities may be worse. At these points there can be extra classes of rational curves. These need not correspond to extra vector moduli. That is, blowing-up the curve of ADE-like singularities may well resolve the "bad point" on the curve too. However, even in this case the extra classes of rational curves can produce more massless states when the gauge group is enhanced.

From what we have said, the homology classes of these extra curves with respect to the homology classes of the rational curves which appear when blowing up the curve of singularities should give the charges of these new states with respect to the Cartan subgroup of the gauge group. That is, we expect these new homology classes to form the weight lattice of the new representation. In this way, we expect the massless hypermultiplets to appear.

Let us apply this approach to our $\mathrm{SO}(32) \times \mathrm{Sp}(k)$ problem. In this case we have a curve of $\mathrm{I}_{12}^{*}$ fibres intersecting a curve of $\mathrm{I}_{2 k}$ fibres transversely at one point. It is at this point of intersection that the new classes can appear. This problem was analyzed in [25, 30]. The result is as follows. Label the homology classes of the exceptional curves in these fibres in 
terms of the Lie algebras they represent as
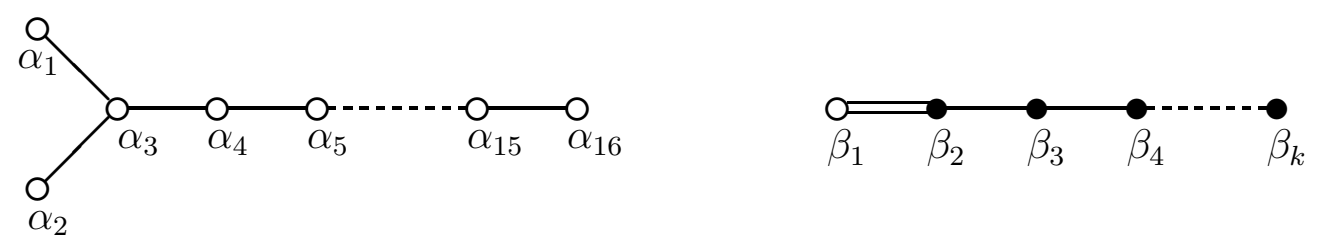

When the curves of $\mathrm{I}_{12}^{*}$ and $\mathrm{I}_{2 k}$ fibres collide transversely, the result at the point of collision according to 25] is an $\mathrm{I}_{12+k}^{*}$ fibre. We label these classes as

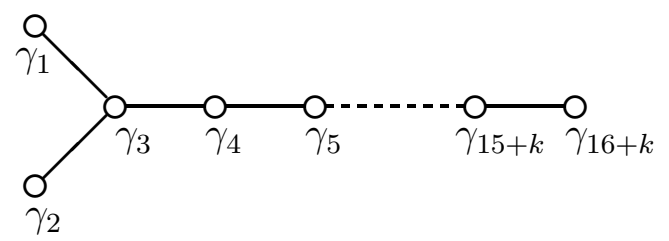

Now we see from [30] that, among the relations, we have

$$
\begin{aligned}
& \alpha_{1}=\gamma_{1} \\
& \alpha_{2}=\gamma_{2} \\
& \beta_{1}=\gamma_{1}+\gamma_{2}+2 \gamma_{3} .
\end{aligned}
$$

That is,

$$
\gamma_{3}=-\frac{1}{2} \alpha_{1}-\frac{1}{2} \alpha_{2}+\frac{1}{2} \beta_{1}
$$

Now $-\frac{1}{2} \alpha_{1}-\frac{1}{2} \alpha_{2}+\frac{1}{2} \beta_{1}$ is a weight of the $(\mathbf{3 2}, \mathbf{2} \boldsymbol{k})$ representation. Furthermore we may obtain the rest of the weights of this representation by adding and subtracting integer multiples of roots. Thus we see that the extra classes introduced by the collision can produce the required extra hypermultiplets. The other $\gamma_{i}$ 's do not introduce any further new weights.

We cannot claim to have shown precisely the existence of every element of the weight lattice here since we need to show that the 2-brane solitons wrap precisely once around each required class on $\mathrm{H}_{2}$. One might try to use some kind of D-brane analysis to prove this. What we can claim however is that if the gauge symmetry exists then we must get a valid weight system for the Lie algebra, and then the weight system of $(\mathbf{3 2}, \mathbf{2} \boldsymbol{k})$ is the minimal such system that contains the rational curve classes we find.

Note we may apply the same techniques to the $E_{8} \times E_{8}$ case as suggested in [9]. Suppose we consider the generic case of an $E_{7}$ gauge group. This involves a curve of III* fibres. One can also see that $\mathrm{I}_{1}$ fibres will cross (non-transversely) this curve at $8-n$ points [9]. Analyzing one of the points of intersection, one may show that a rational curve lives there in one of the weights of the $5 \mathbf{6}$ of $E_{7}$. (We suppress a lengthy algebraic calculation). Note that a hypermultiplet contains both the representation and its complex conjugate of fields. 
Since the $\mathbf{5 6}$ is a quaternionic (or pseudo-real) representation we should really require two to form a single hypermultiplet. We thus expect $\frac{1}{2}(8-n) \mathbf{5 6}$ hypermultiplets in agreement with the index formula [13].

What about the generic case of a curve of $\mathrm{IV}^{*}$ fibres? At first sight, this case would appear to give an $E_{6}$ gauge group. There are $12-2 n$ points along this curve where $\mathrm{I}_{1}$ fibres cross. It turns out that around these collisions there is a $\mathbb{Z}_{2}$ monodromy acting on the $\mathrm{IV}^{*}$ fibre. If $n=6$, or the $12-2 n$ roots pair up in the right way to kill the monodromy, we will have an $E_{6}$ gauge group. Otherwise we claim that the gauge group is actually $F_{4}$ as this is the invariant part of $E_{6}$ divided by a $\mathbb{Z}_{2}$ outer automorphism. ${ }^{2}$ One might expect to see 26's of $F_{4}$ appearing at these crossings but since the weights of the $\mathbf{2 6}$ are actually a subset of the roots, we have no way of deducing this with the methods introduced in this section.

\section{Discussion}

The excellent agreement for the analysis of the $\mathrm{SO}(32)$ heterotic string between the methods shown above and those of [6] appears to be a very strong test of string duality. We also seem to be seeing peculiar relationships between the algebraic geometry of a Calabi-Yau and the properties of vector bundles on a K3 surface. Firstly there are the properties of the enhanced gauge groups themselves, as seen above and in [16, 8, 24, 9] for example. Secondly we have seen how the appearance of rational curves may be linked to massless hypermultiplets.

It is worth emphasizing a difference between the behaviour of generic point-like instantons on the context of the $E_{8} \times E_{8}$ string and the $\mathrm{SO}(32)$ string. For $E_{8} \times E_{8}$, the small instantons have the following properties 10

1. There is no nonperturbative enhancement of the gauge group.

2. There is a massless tensor multiplet for each isolated point-like instanton.

For the $\mathrm{SO}(32)$ case we have [6]

1. There is an $\operatorname{Sp}(1)$ contribution to the nonperturbative part of the gauge group for each isolated point-like instanton.

2. There are no massless tensor multiplets.

3. When point-like instantons coalesce, the gauge group enhances further to $\operatorname{Sp}(k)$.

What happens when point-like instantons of the $E_{8} \times E_{8}$ string coalesce? The elliptic fibration picture shows that blow-ups in the base are required just like the isolated case, and the total number of blow-ups required is the same as the isolated case. The difference is that in this

\footnotetext{
${ }^{2}$ This monodromy was overlooked in [9]. A gauge group of $F_{4}$ for the generic $n=5$ case is also in agreement with the heterotic string picture. See, for example, [24.
} 
case the exceptional divisors intersect. This makes it look very analogous to the coalesced instantons in the $\mathrm{SO}(32)$ string, except that blow-ups are in the base rather than the fibre. We therefore suggest the following interpretation. When $k$ point-like instantons of the $E_{8} \times E_{8}$ string coalesce, extra massless tensor multiplets appear. The tensor multiplets associated to one point have mutual interactions however and expectation values can only be given to $k$ of the them simultaneously.

\section{Acknowledgements}

We would like to thank V. Kaplunovsky, D. Morrison, R. Plesser and H. Tye for useful conversations. The work of the authors is supported by grants from the National Science Foundation.

\section{References}

[1] P. Hořava and E. Witten, Heterotic and Type I String Dynamics from Eleven Dimensions, Nucl. Phys. B460 (1996) 506-524.

[2] E. Witten, String Theory Dynamics in Various Dimensions, Nucl. Phys. B443 (1995) 85-126.

[3] J. Polchinski and E. Witten, Evidence for Heterotic - Type I String Duality, Nucl. Phys. B460 (1996) 525-540.

[4] K. S. Narain, New Heterotic String Theories in Uncompactified Dimensions < 10, Phys. Lett. 169B (1986) 41-46.

[5] P. Ginsparg, On Toroidal Compactification of Heterotic Strings, Phys. Rev. D35 (1987) 648-654.

[6] E. Witten, Small Instantons in String Theory, Nucl. Phys. B460 (1996) 541-559.

[7] E. G. Gimon and J. Polchinski, Consistency Conditions for Orientifolds and D-Manifolds, hep-th/9601038.

[8] D. R. Morrison and C. Vafa, Compactifications of F-Theory on Calabi-Yau Threefolds - I, hep-th/9602114.

[9] D. R. Morrison and C. Vafa, Compactifications of F-Theory on Calabi-Yau Threefolds - II, hep-th/9603161.

[10] N. Seiberg and E. Witten, Comments on String Dynamics in Six Dimensions, hep-th/9603003.

[11] M. Bershadsky et al., Geometric Singularities and Enhanced Gauge Symmetries, to appear. 
[12] C. Vafa and E. Witten, Dual String Pairs With $N=1$ and $N=2$ Supersymmetry in Four Dimensions, hep-th/9507050.

[13] S. Kachru and C. Vafa, Exact Results For N=2 Compactifications of Heterotic Strings, Nucl. Phys. B450 (1995) 69-89.

[14] S. Ferrara, J. Harvey, A. Strominger, and C. Vafa, Second Quantized Mirror Symmetry, Phys. Lett. 361B (1995) 59-65.

[15] P. S. Aspinwall and J. Louis, On the Ubiquity of K3 Fibrations in String Duality, Phys. Lett. 369B (1996) 233-242.

[16] P. S. Aspinwall, Enhanced Gauge Symmetries and Calabi-Yau Threefolds, Phys. Lett. B371 (1996) 231-237.

[17] P. S. Aspinwall and D. R. Morrison, String Theory on K3 Surfaces, hep-th/9404151, to appear in "Essays on Mirror Manifolds 2".

[18] C. Vafa, Evidence for F Theory, hep-th/9602022.

[19] E. Witten, Topological Tools in 10-Dimensional Physics, Int. J. Mod. Phys. A1 (1986) 39-64.

[20] N. Nakayama, On Weierstrass Models, in "Algebraic Geometry and Commutative Algebra in honor of Masayoshi Nagata", pages 405-431, Kinokuniya, 1987.

[21] P. S. Aspinwall, An $N=2$ Dual Pair and a Phase Transition, Nucl. Phys. B460 (1996) $57-76$.

[22] M. Bershadsky, V. Sadov, and C. Vafa, D-Strings and D-Manifolds, hep-th/9510225.

[23] S. Katz, D. R. Morrison, and M. R. Plesser, Enhanced Gauge Symmetry in Type II String Theory, hep-th/9601108.

[24] P. Candelas and A. Font, Duality Between Webs of Heterotic and Type II Vacua, hepth/9603170.

[25] R. Miranda, Smooth Models for Elliptic Threefolds, in R. Friedman and D. R. Morrison, editors, "The Birational Geometry of Degenerations", Birkhauser, 1983.

[26] R. Miranda and U. Persson, On Extremal Rational Elliptic Surfaces, Math. Z. 193 (1986) $537-558$.

[27] S. Chaudhuri and J. Polchinski, Moduli Space of CHL Strings, hep-th/9506048.

[28] W. Fulton and J. Harris, Representation Theory: A First Course, Springer-Verlag, 1991.

[29] A. Strominger, Massless Black Holes and Conifolds in String Theory, Nucl. Phys. B451 (1995) 96-108.

[30] M. Gross, Elliptic Three-folds II: Multiple Fibres, MSRI 1992 preprint, to appear in Trans. of the AMS. 